\title{
Gravitational Collapse of a Shell of Quantized Matter
}

\author{
G.L. Alberghi*, R. Casadio, G.P. Vacca ${ }^{\ddagger}$ and G. Venturi ${ }^{\S}$ \\ Dipartimento di Fisica, Università di Bologna \\ and \\ Istituto Nazionale di Fisica Nucleare, Sezione di Bologna, Italy
}

June 30,2021

\begin{abstract}
The semi-classical collapse, including lowest order back-reaction, of a thin shell of self-gravitating quantized matter is illustrated. The conditions for which selfgravitating matter forms a thin shell are first discussed and an effective Lagrangian for such matter is obtained. The matter-gravity system is then quantized, the semiclassical limit for gravitation is taken and the method of adiabatic invariants is applied to the resulting time dependent matter Hamiltonian. The governing equations are integrated numerically, for suitable initial conditions, in order to illustrate the effect of back-reaction, due to the creation of matter, in slowing down the collapse near the horizon.
\end{abstract}

\section{Introduction}

Much effort has been dedicated to the classical dynamics of collapsing gravitational bodies (see e.g. Refs. [1, 2, 3] for early works and 谏 for a summary of more recent developments). In particular, the collapse of an isotropic homogeneous sphere of dust has been studied using the Arnowitt, Deser and Misner (ADM) construction [5] both on relating the dust associated scalar field to time [6] and including the quantum nature of the collapsing matter through a Born-Oppenheimer approach to the Wheeler-DeWitt (WDW) equation describing the coupled matter-gravity system (see [7, 8, 9] and Refs. therein). Within the latter approach the evolution of quantum matter (dust) was studied both in the adiabatic approximation [10] and on relaxing it 11].

Homogeneous dust in flat space-time without boundaries corresponds to a fluid with (possibly time-dependent) density and zero pressure and can be related to the homogeneous mode of a free massive real scalar field. When dust self-gravitates and is confined into a sphere of finite volume, the metric inside the sphere is a section of a Friedmann universe (in the language of cosmological models) with constant spatial curvature and the

\footnotetext{
*e-mail: alberghi@bo.infn.it

†e-mail: casadio@bo.infn.it

${ }^{\ddagger}$ e-mail: vacca@bo.infn.it

§e-mail: armitage@bo.infn.it
} 
radius of the sphere depends on time [2, 12]. As we have previously indicated [10, 11], using the homogeneous mode of a scalar field to describe a sphere of dust is still plausible as long as one considers spheres whose radius is much greater than the scalar field Compton wave-length. In fact for such a case edge effects are negligible [13].

We have previously, through the use of adiabatic invariants [14] for time dependent Hamiltonians, relaxed the adiabatic approximation and thus allowed for the creation of matter (dust particles). However one will then eventually obtain a non-zero pressure and have deviations from the dust approximation.

Rather than study an isotropic, but inhomogeneous, system it is easier to address the collapse of a gravitating matter shell which we now briefly review, also in order to introduce our notation.

In general the dynamics of a system consisting of self-gravitating matter in a spacetime manifold of 4-volume $V$ with boundary $B$ can be derived from the Einstein-Hilbert action with a matter term

$$
S=\int_{V} d^{4} x \sqrt{-g}\left[\frac{\mathcal{R}^{(4)}}{16 \pi G}+\mathcal{L}_{M}\right]+\frac{1}{8 \pi G} \int_{B} d^{3} x \sqrt{-\tilde{g}} \mathcal{K}=\int L d \tau,
$$

where $G$ is Newton's constant, $g$ the determinant of the 4-dimensional metric, $\mathcal{R}^{(4)}$ the Ricci scalar, $\mathcal{L}_{M}$ the Lagrangian density for matter and $\mathcal{K}$ the trace of the extrinsic curvature of the boundary $B$ on which a 3 -dimensional metric with determinant $\tilde{g}$ is induced.

When matter is localized on an isotropic shell, we can introduce an adapted radial coordinate $r$ such that the shell is parametrized by $r_{i n} \leq r \leq r_{\text {out }}$. Then the external geometry is uniquely determined by Birkhoff's theorem to be given by 15

$$
d s_{\text {in (out) }}^{2}=-A_{\text {in (out) }} d t^{2}+A_{\text {in (out) }}^{-1} d r^{2}+r^{2} d \Omega^{2},
$$

where $A_{i n}=1$ for $r<r_{i n}$, corresponding to a Minkowski metric inside the shell, and $A_{\text {out }}=1-2 G M / r$ for $r>r_{\text {out }}$, corresponding to a Schwarzschild metric of ADM mass $M$ outside the shell.

If the Schwarzschild radius of the shell, $R_{H}=2 G M$, is much greater than the thickness $d \sim r_{\text {out }}-r_{\text {in }}$ of the shell (as we shall show in the next section is true in our case) one can consider the "thin limit" $d / r_{i n} \rightarrow 0$ (with fixed matter proper energy), define $R \equiv\left(r_{\text {in }}+r_{\text {out }}\right) / 2$ and the Lagrangian assumes the form [16, 17]

$$
\begin{aligned}
L= & -\left[\frac{R \dot{R}}{G} \tanh ^{-1}\left(\frac{\dot{R}}{\beta}\right)\right]_{\text {out }}^{\text {in }}+\frac{R}{G}\left(\beta_{\text {in }}-\beta_{\text {out }}\right)-m \\
= & -\frac{R \dot{R}}{G}\left[\tanh ^{-1}\left(\frac{\dot{R}}{\sqrt{1+\dot{R}^{2}}}\right)-\tanh ^{-1}\left(\frac{\dot{R}}{\sqrt{1-\frac{2 G M}{R}+\dot{R}^{2}}}\right)\right]+ \\
& +\frac{R}{G}\left(\sqrt{1+\dot{R}^{2}}-\sqrt{1-\frac{2 G M}{R}+\dot{R}^{2}}\right)-m
\end{aligned}
$$

where $\beta_{\text {in (out) }}=\sqrt{A_{\text {in }(\text { out })}+\dot{R}^{2}},[a]_{\text {out }}^{\text {in }} \equiv a\left(r_{\text {in }}\right)-a\left(r_{\text {out }}\right)$ for any function $a(r)$ and a dot denotes the derivative with respect to the proper time $\tau\left(d \tau=A^{1 / 2} d t\right)$. The quantity $m$ 
is the volume integral of the matter Hamiltonian density and is equal to the proper energy of matter.

The next step is the construction of the Hamiltonian,

$$
\begin{aligned}
H & =P_{R} \dot{R}-L=-\frac{R}{G}\left(\beta_{\text {in }}-\beta_{\text {out }}\right)+m \\
& =m-\frac{\sqrt{2} R}{G}\left[1-\frac{G M}{R}-\sqrt{1-\frac{2 G M}{R}} \cosh \left(\frac{G P_{R}}{R}\right)\right]^{1 / 2}
\end{aligned}
$$

with

$$
P_{R} \equiv \frac{\partial L}{\partial \dot{R}}=-\frac{R}{G}\left[\tanh ^{-1}\left(\frac{\dot{R}}{\beta}\right)\right]_{\text {out }}^{\text {in }} .
$$

It is a general feature of gravitational minisuperspace systems that the equation of motion is obtained on imposing the constraint $H=0$, which expresses the invariance of the action under a time reparametrization. In our case the equation of motion takes the form [3]

$$
\sqrt{1+\dot{R}^{2}}-\sqrt{1-\frac{2 G M}{R}+\dot{R}^{2}}=\frac{G m}{R} .
$$

By squaring twice this expression becomes

$$
\dot{R}^{2}-\frac{G M}{R}-\frac{G^{2} m^{2}}{4 R^{2}}=\frac{M^{2}}{m^{2}}-1 .
$$

In the next section we consider gravitation with a minimally coupled scalar field and study under what conditions one obtains a collapsing shell-like solution. Once this has been achieved, we shall employ the ADM formalism in the Born-Oppenheimer approach to the WDW equation as was done for the sphere [10, 11] and examine the effect of backreaction due to particle creation on the shell during the collapse (section 3). This of course is tantamount to relaxing the adiabatic approximation. Lastly in section 1 our results are summarized and discussed.

\section{The shell structure}

The purpose of this section is to illustrate how one can describe a collapsing matter shell in terms of a scalar field. In particular we shall investigate the conditions under which it is possible to neglect the thickness of the shell and use the thin limit Hamiltonian in Eq. (1.4).

Let us begin by considering an exterior Schwarzschild metric as given in Eq. (1.2) with $A_{\text {out }}$. The action for a massive scalar field $\Phi$ on such a background is

$$
S=\frac{1}{2} \int d t d r r^{2}\left[\left(1-\frac{2 G M}{r}\right)^{-1}\left|\frac{d \Phi}{d t}\right|^{2}-\left(1-\frac{2 G M}{r}\right)\left|\frac{d \Phi}{d r}\right|^{2}-\mu^{2}|\Phi|^{2}\right]
$$


where $\mu$ is the inverse Compton wave-length of the scalar particle described by the field. The associated Klein-Gordon equation satisfied by a spherically symmetric (s-wave) scalar field solution $\Phi(r, t)=\varphi(r) e^{-i \omega t}$ corresponding to a collapse is

$$
\left[-\frac{d}{d r}\left(r^{2}-2 G M r\right) \frac{d}{d r}+\mu^{2} r^{2}-\frac{\omega^{2} r^{3}}{r-2 G M}\right] \varphi=0 .
$$

On taking $r \gg R_{H}$, one obtains

$$
\left[-\frac{d^{2}}{d r^{2}}-\frac{2}{r} \frac{d}{d r}+\left(\mu^{2}-\omega^{2}\right)+\frac{2 G M}{r}\left(\mu^{2}-2 \omega^{2}\right)\right] \varphi=0,
$$

which of course resembles the hydrogen atom case.

The classical limit for the Coulomb case has been studied in detail [18. In particular for large principal quantum number $n$ and angular momentum $l=n-1$ one obtains a probability distribution strongly peaked on classical circular orbits. For the case of a collapse ( $n$ large and $l=0$ ) the probability distribution resembles a spherically symmetric collection of degenerate ellipses. This is analogous to the harmonic oscillator where for large quantum numbers the position probability density similarly approaches that for a classical oscillator of the same energy [19]. This naturally suggests, in analogy with the harmonic oscillator, that one may consider a coherent state, that is a wave packet that will describe the collapse without spreading, at least for a sufficiently large period of time. Unfortunately for the hydrogen atom such a construction has not yet been successfully done (see e.g. [20, 21]).

An alternative way is to construct a shell composed of a suitable number of (isotropic $l=0)$ particles, bound together by gravitational interaction, whose mean position will follow the desired classical collapse. In order to illustrate such an approach let us first consider the semi-classical (WKB) limit in Eq. (2.3) by setting $\varphi \sim e^{i S / \hbar}$. Retaining terms to lowest order in $\hbar$ one has

$$
\left(\frac{d S}{d r}\right)^{2}+\hbar^{2}\left(\mu^{2}-\omega^{2}\right)+\frac{2 G M}{r} \hbar^{2}\left(\mu^{2}-2 \omega^{2}\right)=0 .
$$

On setting $(d S / d r)^{2}=p^{2}\left(\ll \hbar^{2} \mu^{2}\right)$ one obtains

$$
\frac{p^{2}}{2(\hbar \mu)}-\frac{G M(\hbar \mu)}{r}=\hbar^{2} \frac{\left(\omega^{2}-\mu^{2}\right)}{2(\hbar \mu)},
$$

which for $M=(\hbar \mu) / 2$ is just the Newtonian approximation to the equation of motion for the collapse of a self-gravitating shell made of one scalar particle in s-wave with mass $\hbar \mu$ and potential energy $G(\hbar \mu)^{2} / 2 r$ (see Eq. (1.7)).

The r.h.s. of Eq. (2.5) is the (non-relativistic) energy for the motion of a particle in a gravitational field. We can generalize this to the case of $N$ microshells, each one made of one s-wave particle with the same rest mass $\hbar \mu$, constituting a self-gravitating macroshell whose mean radius satisfies a collapse equation similar to that for a shell of total mass $\hbar \mu N$. We shall see that the essential difference with respect to one shell is that the $N$ microshells form a radially localized bound state corresponding to a self-gravitating macroshell of finite thickness. 
Let us start by considering the two microshell case. Each microshell, with mass $\hbar \mu$ and radial position $r_{i}$, has a potential energy which depends on the self-interaction plus the interaction with the inner microshell (if present). Hence the total potential energy can be written as

$$
V\left(r_{1}, r_{2}\right)=-\frac{G(\hbar \mu)^{2}}{2 r_{1}}-\frac{G(\hbar \mu)^{2}}{2 r_{2}}-G(\hbar \mu)^{2}\left[\frac{\theta\left(r_{1}-r_{2}\right)}{r_{1}}+\frac{\theta\left(r_{2}-r_{1}\right)}{r_{2}}\right],
$$

where $\theta$ is the step function. Since we are interested in a thin macroshell we introduce the mean radius coordinate $R=\left(r_{1}+r_{2}\right) / 2$ and the relative coordinate $r=r_{1}-r_{2}$ with $|r| \ll R$. In this approximation it is possible to derive for the potential energy the following expression

$$
V(R, r)=-\frac{G(2 \hbar \mu)^{2}}{2 R}+\frac{G(\hbar \mu)^{2}}{2 R^{2}}|r| .
$$

For realistic values of the total mass of the macroshell (which means that if we wish to consider physical quanta of mass $\hbar \mu$ we must go to a many particle description with more particles on each microshell) the bound state has a characteristic time of oscillation which is several orders of magnitude smaller than the time needed to have a significant variation of the centre of mass position. For this reason it is possible to factorize the mean radius motion and study the quantum bound state adiabatically with respect to the slowly varying $R(t)$. An interesting fact worth noting is the (slow) increase of the coupling during the collapse (since $R$ decreases) which implies that the average shell thickness will decrease. For $R$ constant the quantum problem for the relative $r$ degree of freedom is well known and can be solved, with the spectrum of the allowed energy excitations being given in terms of the zeros of the Airy function or of its derivative. It is also possible to check that a semi-classical (WKB) analysis gives a good estimate of the energy levels, even for low quantum numbers.

A similar conclusion to the above can be obtained in a classical general relativistic framework by using the junction conditions [3] and taking the Newtonian limit. For a system of two non-crossing shells one has

$$
\begin{aligned}
& \left(\frac{d r_{1}}{d t}\right)^{2}=\left(1-\frac{2 G M_{1}}{r_{1}}\right)\left[-1+\frac{M_{1}^{2}}{m_{1}^{2}}+\frac{G M_{1}}{r_{1}}+\frac{G^{2} m_{1}^{2}}{4 r_{1}^{2}}\right] \\
& \left(\frac{d r_{2}}{d t}\right)^{2}=\left(1-\frac{2 G M_{1}}{r_{2}}\right)\left[-1+\frac{\left(M_{2}-M_{1}\right)^{2}}{m_{2}^{2}}+\frac{G\left(M_{1}+M_{2}\right)}{r_{2}}+\frac{G^{2} m_{2}^{2}}{4 r_{2}^{2}}\right],
\end{aligned}
$$

where $m_{1}, m_{2}$ are the proper masses of the shells positioned at $r_{1}<r_{2}, M_{1}$ and $t$ are the Schwarzschild mass and time coordinate for the metric between the two shells while $M_{2}$ is the Schwarzschild mass for the metric outside the outer shell. Since we wish to analyze the interaction between the shells in the Newtonian limit we neglect the terms proportional to $G^{2}$ in the above. On taking $m_{1}=m_{2}=\hbar \mu$ one may sum (to this order) the two junction equations, multiply the result by $\hbar \mu / 2$ and include the two position independent terms in the total energy $E$. One then has, for $r_{1}<r_{2}$,

$$
E=\frac{1}{2} \hbar \mu\left[\left(\frac{d r_{1}}{d t}\right)^{2}+\left(\frac{d r_{2}}{d t}\right)^{2}\right]-\frac{G(\hbar \mu)^{2}}{2 r_{1}}-\frac{3}{2} \frac{G(\hbar \mu)^{2}}{r_{2}}
$$


An analogous term is easily obtained for the case $r_{1}>r_{2}$ and finally the potential in Eq. (2.6) is re-obtained.

If one wishes to consider a collapse approaching the horizon it is convenient to use the proper time of one of the two shells in Eq. (2.8). In fact it is easy to check that on the horizon the ratio of the proper times of the two shells is 1 up to corrections of order $d / R$ which we require to be negligible (see Eq. (2.21) for an estimate of this quantity). This implies that one is also allowed to consider the proper time associated with the mean shell radius.

Let us now examine the more interesting $N$ microshell case, again in the Newtonian limit. One may consider each microshell at a radial position $r_{i}$ and define $r_{i j}=r_{i}-r_{j}$. The full interaction potential can then be derived:

$$
V\left(r_{1}, \ldots, r_{N}\right)=-\sum_{i=1}^{N} \frac{G(\hbar \mu)^{2}}{2 r_{i}}-\sum_{i=1}^{N} \frac{G(\hbar \mu)^{2}}{2 r_{i}} \sum_{j \neq i} \theta\left(r_{i j}\right),
$$

where the first sum refers to the gravitational self-interaction and the second sum to the interparticle interaction. Introducing the mean shell radius $R=\left(\sum_{i} r_{i}\right) / N$ one can write $r_{i}=R-\left(\sum_{j \neq i} r_{j i}\right) / N$ and on using the thin shell approximation $\left|r_{i j}\right| \ll R$ one has

$$
V\left(r_{1}, \ldots, r_{N}\right)=-\frac{G(N \hbar \mu)^{2}}{2 R}+\frac{G(\hbar \mu)^{2}}{2 R^{2}} \sum_{i<j}\left|r_{i j}\right| .
$$

In this expression for the potential energy the first term indicates that the mean shell radius motion follows approximately the Newtonian collapse of an object (the macroshell) of mass $m=N \hbar \mu$ and the second term refers to the gravitational interaction of the internal degrees of freedom of the macroshell (relative positions of the microshells). One may study in the adiabatic approximation the quantum mechanical behaviour of the internal motion of the microshells but the problem is still complex and apparently unsolvable. It corresponds to the case of many particles with a confining potential linearly growing with the interparticle distances. This of course is analogous to the quark confining potential in hadronic physics, however for the latter case the total charge (colour) is zero. Further, due to the bosonic nature of the particles, in our case a condensate should be expected.

At later stages of the collapse, as $R(t)$ varies more rapidly, one expects non-adiabatic effects leading to transitions from the ground to higher (excited) states for some of the constituents of the particle condensate forming the macroshell. Clearly such excited states may decay emitting escaping radiation. However to describe this would mean introducing interaction terms and having an exterior Vaidya metric thus greatly complicating our simple model. The attitude we shall take and implement in the next sections is that once enough particles in the condensate are excited (thus widening the macroshell) they will collectively decay to the ground state by creating additional particles [22]. Thus the macroshell during its collapse will be globally described as a condensate of particles (all in the ground state) whose number varies (increases).

In order to understand how the number $N$ of microshells affects the global internal energy and the thickness of the macroshell we used a many body Hartree approach to evaluate the energy for some trial parameter-dependent vacuum wave function. The average 
Hamiltonian for a fully symmetric state $\prod_{i}^{N} \varphi\left(r_{i}\right)$ is given by

$$
\begin{aligned}
\langle\hat{H}\rangle= & \frac{\hbar^{2}}{2 \hbar \mu} N \int d r\left|\frac{d \varphi(r)}{d r}\right|^{2}-\frac{G(N \hbar \mu)^{2}}{2 R} \\
& +\frac{N(N-1) G(\hbar \mu)^{2}}{4 R^{2}} \int d r d r^{\prime}|\varphi(r)|^{2}\left|r-r^{\prime}\right|\left|\varphi\left(r^{\prime}\right)\right|^{2},
\end{aligned}
$$

where we consider $\varphi(r)=c e^{-\alpha|r-R|^{p}} e^{i k R}$ for the trial vacuum wave function with $c$ a normalization factor and the phase factor describes the mean shell radius behaviour which is approximately free. Let us note that the asymptotic behaviour of the exact wave function for the $N=2$ case is given by $p=3 / 2$. One can verify that minimizing the average Hamiltonian for the relative motion with respect to $\alpha$ leads, independently of $p$, to the following qualitative behaviour

$$
\left\langle\hat{H}_{r e l}\right\rangle \sim \hbar \mu N(N-1)^{2 / 3}\left(\frac{G}{R^{2}}\right)^{2 / 3}
$$

and $p$ only affects the magnitude of the proportionality constant. Further one also obtains an estimate of the width $d$ of the packet

$$
d \sim \alpha^{-1 / p} \sim \frac{1}{\mu}\left(\frac{G(N-1)}{R^{2}}\right)^{-1 / 3} .
$$

A similar behaviour can also be found if one calculates the energy levels semi-classically. Indeed for such a case the potential term may be evaluated by considering the microshells to be well ordered [23] so that the positions $\tilde{r}_{k}=r_{k}-R$ (with respect to the mean shell radius) satisfy $\tilde{r}_{1} \leq \tilde{r}_{2} \leq \ldots \leq \tilde{r}_{N}$ and one will have $r_{k+1, k} \geq 0$ with

$$
\sum_{k=1}^{N} \sum_{j<k}\left|r_{k j}\right|=\sum_{k=1}^{N-1} k(N-k) r_{k+1, k}=2 \sum_{k=1}^{N} k \tilde{r}_{k} .
$$

The $N$ microshell Hamiltonian then becomes

$$
H=\sum_{k=1}^{N} H_{k}=\sum_{k=1}^{N}\left[\frac{1}{2} \hbar \mu \dot{\tilde{r}}_{k}^{2}+\frac{G(\hbar \mu)^{2}}{2 R^{2}}(2 k) \tilde{r}_{k}\right] .
$$

We may now consider the $k$-th microshell whose amplitude of oscillation will be the width $d$ of the shell. Taking a closed cycle for the $k$-th microshell one has a semiclassical quantization condition:

$$
4 \hbar \mu \sqrt{\frac{G \hbar \mu}{R^{2}}(2 k)} \int_{0}^{d}\left(d-\tilde{r}_{k}\right)^{1 / 2} d \tilde{r}_{k}=\left(n+\frac{1}{2}\right) \hbar,
$$

which leads to an energy

$$
E_{k}=\left(\frac{9}{128} \hbar \mu\right)^{1 / 3}\left[\frac{G \hbar \mu}{2 R^{2}}(2 k)\left(n+\frac{1}{2}\right) \hbar\right]^{2 / 3}
$$


and

$$
E=\sum_{k=1}^{N} E_{k} \approx \frac{N^{5 / 3}}{16} \hbar \mu\left[\frac{3}{2} \frac{G}{R^{2}}\left(n+\frac{1}{2}\right) \hbar\right]^{2 / 3}
$$

in agreement with Eq. (2.13). We note that the binding energy increases faster that the microshell number. In particular for a given $n$ the smaller is $R$ and/or the larger is the number $N$ of microshells the smaller is the thickness $d$ of the macroshell.

Using natural units $\hbar=c=1$ one has $G=1 / m_{p l}^{2} \simeq 10^{-38} \mathrm{GeV}^{-2}$ and we can obtain some numerical estimates. If we consider matter quanta with a mass $\hbar \mu=1$ $\mathrm{GeV}$ and a number of quanta $N=10^{51}$ (giving a total mass corresponding to $10^{-6}$ solar masses), the Schwarzschild radius will be $2 G m=10^{13} \mu^{-1} \simeq 0.2 \mathrm{~cm}$ and for a mean shell radius located at $R=10^{8} \mathrm{Gm} \simeq 200 \mathrm{~km}$, one has, from Eq. (2.14), an estimated thickness of the macroshell $d \sim 10^{10} \mu^{-1} \sim 10^{-4} \mathrm{~cm}$. With these numbers the potential energy of the mean radius of the macroshell is $\mathrm{Gm}^{2} /(2 R) \sim 10^{43} \mathrm{GeV}$, while the total internal energy for the other degrees of freedom, assumed to be in the ground state, is $E_{\text {int }} \sim m\left(G N / R^{2}\right)^{2 / 3} \sim 10^{32} \mathrm{GeV}$ (higher occupation numbers would lead to the previously shown $n^{2 / 3}$ behaviour). It is clear from these numbers that the condensatemaintaining mechanism we previously envisaged is plausible: a limited number of excited states can easily supply the energy for the creation of additional particles thus maintaining the condensate.

From the above picture it is clear that, for a wide regime during the collapse, modelling the macroshell in terms of scalar fields confined around the mean shell radius is a good approximation. Thus we shall take for the scalar field describing one of the $N$ particles a form $\Phi(r, t)=e^{-i \mu t} \phi(t) \varphi(r) / \sqrt{\mu}$ with $\varphi(r)$ describing a packet with a width of order $d$ around $R$. This time $\varphi(r)$ will be normalized with the measure $d r$ and not $r^{2} d r$ since we shall consider the dynamics of the field $\phi(t)$ with $R(t)$ a time varying parameter. Then, if one considers a radial wave packet similar to the one used in the Hartree approximation and performs the radial integration in the action (2.1) for the scalar field, one obtains an effective action for $\phi(t)$. In terms of the proper time $\tau$ such effective action is given by

$$
S \approx \frac{1}{2 \mu} \int d \tau R^{2}(\tau)\left[\left(\frac{d \phi}{d \tau}\right)^{2}-\mu^{2} \phi^{2}-\mu^{2}\left(\frac{G N}{R^{2}}\right)^{2 / 3} \phi^{2}\right] .
$$

The last term in the integrand, which is related to the shell thickness, on comparing with the second term, can be safely neglected. Indeed, with the numbers previously given it is suppressed by a factor of $10^{19}$. In the next section we shall use the formalism of second quantization in order to describe the $N$ particle system discussed above.

Lastly we note that, since we shall be interested in the evolution of the shell until it reaches the Schwarzschild radius $R_{H}$, which can be approximated extremely well by $2 \mathrm{Gm}$, we must check that the ratio

$$
\frac{d}{R} \simeq \frac{E_{\text {int }}}{m} \sim\left(\frac{G N}{R^{2}}\right)^{2 / 3}
$$

is still negligible with respect to 1 near $R_{H}$, that is

$$
G N \mu \sim R_{H} \gg 1 / \mu
$$

For the values given above $(\mu=1 \mathrm{GeV})$, we need $N \gg 1 / G \mu^{2} \sim 10^{38}$. 


\section{The quantized shell}

We may now write the WDW equation for the collapsing shell and the scalar field. The corresponding Hamiltonian will be given by Eq. (1.4) with $m$ the average value for $N$ particles of the Hamiltonian for the quantum scalar field obtained from Eq. (2.20). Let us now illustrate this. On expanding the Hamiltonian in Eq. (1.4) in $G$ and retaining terms up to order $G$ one has

$$
H=\frac{\mu}{2}\left[\frac{\pi_{\phi}^{2}}{R^{2}}+R^{2} \phi^{2}\right]-M+\frac{P_{R}^{2}}{2 M}-\frac{G M^{2}}{2 R} \equiv H_{M}+H_{G},
$$

where $\pi_{\phi}=R^{2} \dot{\phi} / \mu$ and $P_{R}=M \dot{R} / \sqrt{1+\dot{R}^{2}}$. Canonical quantization then leads to the WDW equation

$$
\left[\frac{\mu}{2}\left(-\frac{\hbar^{2}}{R^{2}} \frac{\partial^{2}}{\partial \phi^{2}}+R^{2} \phi^{2}\right)-M-\frac{\hbar^{2}}{2 M} \frac{\partial^{2}}{\partial R^{2}}-\frac{G M^{2}}{2 R}\right] \Psi(R, \phi)=0,
$$

and there is no operator ordering ambiguity.

\subsection{Born-Oppenheimer approach}

We express the function $\Psi$ in the form $\Psi(R, \phi)=\psi(R) \chi(\phi, R)$ which allows us to obtain the WDW equation for the gravitational part [9]

$$
\begin{gathered}
{\left[-\left(M+\frac{G M^{2}}{2 R}+\frac{\hbar^{2}}{2 M} \frac{\partial^{2}}{\partial R^{2}}\right)+\frac{1}{2\langle\tilde{\chi} \mid \tilde{\chi}\rangle}\left\langle\tilde{\chi}\left|\left(\mu \frac{\hat{\pi}_{\phi}^{2}}{R^{2}}+\mu R^{2} \phi^{2}\right)\right| \tilde{\chi}\right\rangle\right] \tilde{\psi}} \\
\equiv\left[\hat{H}_{G}+\left\langle H_{M}\right\rangle\right] \tilde{\psi}=\frac{\hbar^{2}}{2 M} \frac{1}{\langle\tilde{\chi} \mid \tilde{\chi}\rangle}\left\langle\tilde{\chi}\left|\frac{\partial^{2}}{\partial R^{2}}\right| \tilde{\chi}\right\rangle \equiv \frac{\hbar^{2}}{2 M}\left\langle\frac{\partial^{2}}{\partial R^{2}}\right\rangle \tilde{\psi}
\end{gathered}
$$

where we have defined a scalar product

$$
\langle\chi \mid \chi\rangle \equiv \int d \phi \chi^{*}(\phi, R) \chi(\phi, R)
$$

and set

$$
\psi=e^{-i \int^{R} A\left(R^{\prime}\right) d R^{\prime}} \tilde{\psi} \quad \chi=e^{+i \int^{R} A\left(R^{\prime}\right) d R^{\prime}} \tilde{\chi}
$$

with

$$
A \equiv-\frac{i}{\langle\chi \mid \chi\rangle}\left\langle\chi\left|\frac{\partial}{\partial R}\right| \chi\right\rangle \equiv-i\left\langle\frac{\partial}{\partial R}\right\rangle
$$

Further, we also obtain the equation for the matter field

$$
\tilde{\psi}\left[\hat{H}_{M}-\left\langle\hat{H}_{M}\right\rangle\right] \tilde{\chi}+\frac{\hbar^{2}}{2 M}\left(\frac{\partial \tilde{\psi}}{\partial R}\right)\left(\frac{\partial \tilde{\chi}}{\partial R}\right)=\frac{\hbar^{2}}{2 M} \tilde{\psi}\left[\left\langle\frac{\partial^{2}}{\partial R^{2}}\right\rangle-\frac{\partial^{2}}{\partial R^{2}}\right] \tilde{\chi}
$$


As yet there is no time parameter, however, on neglecting the r.h.s. of Eq. (3.3), one may introduce a time variable by taking the semi-classical (WKB) approximation for the gravitational wave function $\tilde{\psi}$,

$$
\tilde{\psi} \simeq\left(\frac{\partial S_{e f f}}{\partial R}\right)^{-1 / 2} e^{\frac{i}{\hbar} S_{e f f}}
$$

where the effective action $S_{\text {eff }}$ satisfies the Hamilton-Jacobi equation

$$
\left\langle\hat{H}_{M}\right\rangle+H_{G}=0
$$

associated with the l.h.s. of Eq. (3.3), and in the non-relativistic limit, $\dot{R} \ll 1$, it is given by

$$
S_{\text {eff }}=\frac{1}{2} \int d \tau\left[2 M+M \dot{R}^{2}+\frac{G M^{2}}{R}-2\left\langle\hat{H}_{M}\right\rangle\right],
$$

where $\left\langle\hat{H}_{M}\right\rangle$ is evaluated on the semi-classical trajectory where $\tilde{\psi}$ has support.

The explicit expression of Eq. (3.9),

$$
\dot{R}^{2}=\frac{2}{M}\left(M-\left\langle\hat{H}_{M}\right\rangle\right)+\frac{G M}{R},
$$

may be compared with the classical Eq. (1.7). The two equations lead to the same evolution if

$$
\left\langle\hat{H}_{M}\right\rangle=m
$$

and

$$
\begin{aligned}
& \frac{G^{2} m^{2}}{4 R^{2}} \ll \frac{G M}{R} \Rightarrow \frac{G m^{2}}{R M} \ll 1 \\
& \frac{M^{2}}{m^{2}}-1 \simeq \frac{2}{M}(M-m) \Rightarrow m / M \simeq 1 .
\end{aligned}
$$

With the above conditions we can obtain as our equation of motion for $R$

$$
\dot{R}^{2}-\frac{G M}{R}=\frac{M^{2}}{m^{2}}-1,
$$

which is just the equation for radial geodesics in Schwarzschild space-time. In particular, on comparing Eq. (3.14) with the standard notation for a radial trajectory with geodesic energy $E$ in a Schwarzschild space-time with mass parameter $\tilde{M}$ (see e.g. [12]),

$$
\dot{R}^{2}=\frac{2 G \tilde{M}}{R}-\left(1-E^{2}\right)
$$

we find $\tilde{M}=M / 2$ and $E=M / m$. Hence the shell evolves as a particle in a Schwarzschild manifold of total energy $M / 2$, that is half of the total energy of the shell. The solutions to Eq. (3.14) are known. Here we only recall for later reference the case $m=M$, corresponding to the separatrix between bound orbits and scattering trajectories:

$$
R_{c}(\tau)=R_{0}\left[1-\frac{3}{2} \sqrt{\frac{G M}{R_{0}^{3}}} \tau\right]^{2 / 3}
$$


where $R_{0} \equiv R(0)$.

The proper time variable associated to the WKB gravitational wave function is given by the relation

$$
\begin{aligned}
\frac{\partial \tilde{\psi}}{\partial R} \frac{\partial}{\partial R} & \simeq\left[\frac{i}{\hbar} \frac{\partial S_{e f f}}{\partial R}-\frac{1}{2}\left(\frac{\partial S_{e f f}}{\partial R}\right)^{-1} \frac{\partial^{2} S_{e f f}}{\partial R^{2}}\right] \tilde{\psi} \frac{\partial}{\partial R} \\
& \equiv-\frac{i}{\hbar} M \tilde{\psi} \frac{\partial}{\partial \tau}-\frac{1}{2}\left(\frac{\partial S_{e f f}}{\partial R}\right)^{-1} \frac{\partial^{2} S_{e f f}}{\partial R^{2}} \tilde{\psi} \frac{\partial}{\partial R}
\end{aligned}
$$

If the r.h.s. of Eq. (3.7) and the second term in Eq. (3.17) are negligible, one obtains a Schrödinger equation for the matter wave-function $\chi_{s}$,

$$
i \hbar \frac{\partial \chi_{s}}{\partial \tau}=\frac{\mu}{2}\left[-\frac{\hbar^{2}}{R^{2}} \frac{\partial^{2}}{\partial \phi^{2}}+R^{2} \phi^{2}\right] \chi_{s}=\hat{H}_{M} \chi_{s}
$$

where we have scaled the dynamical phase

$$
\chi_{s} \equiv \tilde{\chi} \exp \left\{-\frac{i}{\hbar} \int^{\tau}\left\langle\hat{H}_{M}\left(\tau^{\prime}\right)\right\rangle d \tau^{\prime}\right\}
$$

\subsection{Adiabatic invariants}

In order to study quantum systems with explicitly time-dependent Hamiltonians we can use the method of invariants [14]. Given a Hamiltonian $\hat{H}_{M}(\tau)$, a Hermitian operator $\hat{I}(\tau)$ is an invariant if it satisfies

$$
i \hbar \frac{\partial \hat{I}(\tau)}{\partial \tau}+\left[\hat{I}(\tau), \hat{H}_{M}(\tau)\right]=0 .
$$

The general solution $\chi_{s}(\tau)$ to the Schrödinger equation

$$
i \hbar \frac{\partial \chi_{s}(\tau)}{\partial \tau}=\hat{H}_{M}(\tau) \chi_{s}(\tau)
$$

can then be written in the form

$$
|\chi, \tau\rangle_{I s}=\sum_{n} C_{n} e^{i \varphi_{n}(\tau)}|n, \tau\rangle_{I},
$$

where $|n, \tau\rangle_{I}$ is an eigenvector of $\hat{I}(\tau)$ with time-independent eigenvalue $\lambda_{n}$ and $C_{n}$ are complex coefficients. The phase $\varphi_{n}(\tau)$ is given by the sum of the geometrical and dynamical phases associated respectively to Eqs. (3.6) and (3.19),

$$
\varphi_{n}(\tau)=\frac{i}{\hbar} \int_{\tau_{0}}^{\tau}{ }_{I}\left\langle n, \tau^{\prime}\left|\hbar \partial_{\tau^{\prime}}+i \hat{H}_{M}\left(\tau^{\prime}\right)\right| n, \tau^{\prime}\right\rangle_{I} d \tau^{\prime}
$$

The Hamiltonian in Eq. (3.18) describes an harmonic oscillator of fixed frequency $\mu$ and variable mass $R^{2} / \mu$. Thus we can introduce the linear (non-Hermitian) invariant

$$
\hat{I}_{b}(\tau) \equiv e^{i \Theta(\tau)} \hat{b}(\tau)
$$


with the phase $\Theta(\tau)$ given by

$$
\Theta(\tau)=\int_{\tau_{0}}^{\tau} d \tau^{\prime} \frac{\mu}{R^{2}\left(\tau^{\prime}\right) x^{2}\left(\tau^{\prime}\right)}
$$

and

$$
\hat{b}(\tau) \equiv \frac{1}{\sqrt{2 \hbar}}\left[\frac{\hat{\phi}}{x}+i\left(x \hat{\pi}_{\phi}-\dot{x} \frac{R^{2}}{\mu} \hat{\phi}\right)\right] .
$$

The function $x(\tau)$ is a solution of

$$
\ddot{x}+2 \dot{x} \frac{\dot{R}}{R}+\mu^{2} x=\frac{\mu^{2}}{R^{4} x^{3}},
$$

with suitable initial conditions.

The system admits an invariant ground state (vacuum) $|0, \tau\rangle_{b}$ defined by

$$
\hat{I}_{b}(\tau)|0, \tau\rangle_{b}=0
$$

from which one can define a basis of eigenstates $\mathcal{B}=\left\{|n, \tau\rangle_{b}\right\}$,

$$
\begin{aligned}
|n, \tau\rangle_{b s} & \equiv \frac{\left(\hat{I}_{b}^{\dagger}\right)^{n}}{\sqrt{n !}}|0, \tau\rangle_{b s} \\
& =e^{-i n \Theta} \frac{\left(\hat{b}^{\dagger}\right)^{n}}{\sqrt{n !}}|0, \tau\rangle_{b s} \\
& =e^{i\left(\varphi_{0}-n \Theta\right)} \frac{\left(\hat{b}^{\dagger}\right)^{n}}{\sqrt{n !}}|0, \tau\rangle_{b}=e^{i \varphi_{n}(\tau)}|n, \tau\rangle_{b}
\end{aligned}
$$

where $\varphi_{0}$ is arbitrary and can be replaced by $\Theta / 2$, and

$$
\begin{gathered}
\hat{b}|n, \tau\rangle_{b}=\sqrt{n}|n-1, \tau\rangle_{b} \\
\hat{b}^{\dagger}|n, \tau\rangle_{b}=\sqrt{n+1}|n+1, \tau\rangle_{b} .
\end{gathered}
$$

Since $\left[\hat{b}, \hat{b}^{\dagger}\right]=1$ we will refer to $\hat{b}^{\dagger}$ and $\hat{b}$ as the invariant creation and annihilation operators. We can then introduce the Hermitian quadratic invariant operator

$$
\hat{I}_{c} \equiv\left(\hat{I}_{b}^{\dagger} \hat{I}_{b}+\frac{1}{2}\right)=\hbar\left(\hat{b}^{\dagger} \hat{b}+\frac{1}{2}\right)
$$

in which $\hat{b}^{\dagger} \hat{b}$ is the invariant number operator.

We also define the particle creation and annihilation operators $\hat{a}^{\dagger}$ and $\hat{a}$ (with $\left[\hat{a}^{\dagger}, \hat{a}\right]=$ 1) as

$$
\begin{aligned}
\hat{a}(\tau) & =\frac{R}{\sqrt{2 \hbar}}\left(\hat{\phi}+i \frac{\hat{\pi}_{\phi}}{R^{2}}\right) \\
\hat{a}^{\dagger}(\tau) & =\frac{R}{\sqrt{2 \hbar}}\left(\hat{\phi}-i \frac{\hat{\pi}_{\phi}}{R^{2}}\right)
\end{aligned}
$$


in terms of which the matter Hamiltonian operator can be expressed as

$$
\hat{H}_{M}=\hbar \mu\left(\hat{N}+\frac{1}{2}\right)
$$

where $\hat{N} \equiv \hat{a}^{\dagger} \hat{a}$ is the particle number operator which counts the number of quanta of the scalar field $\phi$. The corresponding vacuum state $|0, \tau\rangle_{a}$ is defined by

$$
\hat{a}|0, \tau\rangle_{a}=0
$$

and a complete set of eigenstates can be given as $\mathcal{A}=\left\{|n, \tau\rangle_{a}\right\}$,

$$
|n, \tau\rangle_{a} \equiv \frac{\left(\hat{a}^{\dagger}\right)^{n}}{\sqrt{n !}}|0, \tau\rangle_{a}
$$

with

$$
\begin{gathered}
\hat{a}|n, \tau\rangle_{a}=\sqrt{n}|n-1, \tau\rangle_{a} \\
\hat{a}^{\dagger}|n, \tau\rangle_{a}=\sqrt{n+1}|n+1, \tau\rangle_{a} .
\end{gathered}
$$

The two Fock basis $\mathcal{A}$ and $\mathcal{B}$ are related. On using

$$
\begin{gathered}
\hat{\phi}=\sqrt{\frac{\hbar}{2}} x\left(\hat{b}+\hat{b}^{\dagger}\right) \\
\hat{\pi}_{\phi}=\sqrt{\frac{\hbar}{2}}\left[\frac{i}{x}\left(\hat{b}^{\dagger}-\hat{b}\right)+\dot{x} \frac{R^{2}}{\mu}\left(\hat{b}^{\dagger}+\hat{b}\right)\right],
\end{gathered}
$$

we obtain

$$
\left\{\begin{array} { l } 
{ \hat { a } = B ^ { * } \hat { b } + A ^ { * } \hat { b } ^ { \dagger } } \\
{ \hat { a } ^ { \dagger } = B \hat { b } ^ { \dagger } + A \hat { b } }
\end{array} \quad \text { or } \quad \left\{\begin{array}{l}
\hat{b}=B \hat{a}-A^{*} \hat{a}^{\dagger} \\
\hat{b}^{\dagger}=B^{*} \hat{a}^{\dagger}-A \hat{a}
\end{array}\right.\right.
$$

where

$$
\begin{aligned}
& A(\tau)=\frac{1}{2} R\left(x-\frac{1}{R^{2} x}-i \frac{\dot{x}}{\mu}\right) \\
& B(\tau)=\frac{1}{2} R\left(x+\frac{1}{R^{2} x}-i \frac{\dot{x}}{\mu}\right)
\end{aligned}
$$

are the Bogoliubov coefficients. The two basis $\mathcal{A}$ and $\mathcal{B}$ will coincide for $\tau=\tau_{0}$ if

$$
\hat{b}\left(\tau_{0}\right)=\hat{a}\left(\tau_{0}\right) \quad \Rightarrow \quad \mu \hat{I}_{c}\left(\tau_{0}\right)=\hat{H}_{M}\left(\tau_{0}\right),
$$

that is $A\left(\tau_{0}\right)=0$ and $B\left(\tau_{0}\right)=1$, which means that for the function $x(\tau)$ we must require

$$
\left\{\begin{array}{l}
x\left(\tau_{0}\right)=1 / R \\
\dot{x}\left(\tau_{0}\right)=0 .
\end{array}\right.
$$

In the following we shall take $\tau_{0}=0$ so that the adiabatic approximation holds at the beginning of the collapse. 
If we define $\sigma \equiv R x$, Eq. (3.27) takes the form

$$
\frac{\ddot{\sigma}}{\mu^{2}}+\Omega^{2} \sigma=\frac{1}{\sigma^{3}}
$$

which is the equation for a harmonic oscillator with time-dependent frequency $\Omega$,

$$
\Omega^{2}=1+\frac{\ddot{R}}{\mu^{2} R^{2}} .
$$

On using the equation of motion (3.14) one has

$$
\Omega^{2} \simeq 1+\frac{G M}{2 \mu^{2} R^{3}} \equiv 1+\frac{\delta^{2}}{2} \frac{R_{0}^{3}}{R^{3}},
$$

where $\delta$ (also suggested by Eq. (3.16)) plays the role of a parameter measuring nonadiabaticity since for $\delta \rightarrow 0$ the adiabatic limit is recovered. On expanding in $\delta$, an approximate analytic solution to Eq. 3.42 is given by 24]

$$
\sigma \simeq \Omega^{-1 / 2}=1-\frac{\delta^{2}}{8} \frac{R_{0}^{3}}{R^{3}}
$$

Then

$$
x=\frac{\sigma}{R} \simeq \frac{1}{R}\left[1-\frac{\delta^{2}}{8} \frac{R_{0}^{3}}{R^{3}}\right]
$$

and

$$
\Theta(\tau) \simeq \mu \int_{0}^{\tau} d \tau^{\prime}\left[1+\frac{\delta^{2}}{4} \frac{R_{0}^{3}}{R^{3}}\right] .
$$

The introduction of the eigenstates of adiabatic invariants allows us to examine the particle production induced by the evolution of the radius of the shell. As we have seen previously, in order to have a physical shell we must consider a large number of particles. Thus let us consider a linear combination of eigenstates of the invariant number operator defined in Eq. (3.29),

$$
\left|N_{\lambda}, \tau\right\rangle_{b s}=\frac{1}{\sqrt{2 \lambda}} \sum_{n=N-\lambda}^{N+\lambda}|n, \tau\rangle_{b s}
$$

with $1 \ll \lambda \ll N$. Then we can evaluate the quantities of interest as expectation values for this state. On defining $\langle\hat{O}\rangle \equiv{ }_{b s}\left\langle N_{\lambda}, \tau|\hat{O}| N_{\lambda}, \tau\right\rangle_{b s}$ we obtain (up to corrections of $\mathcal{O}(\lambda / N))$

$$
\begin{aligned}
\langle\phi\rangle & \simeq \sqrt{2 \hbar N} x \cos \Theta \\
\left\langle\pi_{\phi}\right\rangle & \simeq \sqrt{2 \hbar N}\left[\frac{\dot{x} R^{2}}{\mu} \cos \Theta-\frac{1}{x} \sin \Theta\right]
\end{aligned}
$$


and

$$
\begin{aligned}
\left\langle(\Delta \hat{\phi})^{2}\right\rangle & \simeq \frac{\hbar}{2} x^{2} \\
\left\langle\left(\Delta \hat{\pi}_{\phi}\right)^{2}\right\rangle & \simeq \frac{\hbar}{2}\left(\frac{1}{x^{2}}+\frac{R^{4}}{\mu^{2}} \dot{x}^{2}\right),
\end{aligned}
$$

which lead to an uncertainty relation

$$
\left\langle(\Delta \hat{\phi})^{2}\right\rangle^{1 / 2}\left\langle\left(\Delta \hat{\pi}_{\phi}\right)^{2}\right\rangle^{1 / 2} \simeq \frac{\hbar}{2} \sqrt{1+\frac{R^{4}}{\mu^{2}} x^{2} \dot{x}^{2}} .
$$

On substituting the explicit expression for $x$ and $\dot{x}$ from Eq. 3.46 we obtain

$$
\begin{aligned}
\left\langle(\Delta \hat{\phi})^{2}\right\rangle & \simeq \frac{\hbar}{2} \frac{1}{R^{2}}\left(1-\frac{\delta^{2}}{4} \frac{R_{0}^{3}}{R^{3}}\right) \\
\left\langle\left(\Delta \hat{\pi}_{\phi}\right)^{2}\right\rangle & \simeq \frac{\hbar}{2}\left[R^{2}\left(1+\frac{\delta^{2}}{4} \frac{R_{0}^{3}}{R^{3}}\right)+\frac{\dot{R}^{2}}{\mu^{2}}\left(1-\delta^{2} \frac{R_{0}^{3}}{R^{3}}\right)\right],
\end{aligned}
$$

and

$$
\left\langle(\Delta \hat{\phi})^{2}\right\rangle^{1 / 2}\left\langle\left(\Delta \hat{\pi}_{\phi}\right)^{2}\right\rangle^{1 / 2} \simeq \frac{\hbar}{2}\left[1+\frac{\dot{R}^{2}}{\mu^{2} R^{2}}\left(1-\frac{5}{4} \delta^{2} \frac{R_{0}^{3}}{R^{3}}\right)\right]^{1 / 2} .
$$

We also find

$$
\left\langle\hat{I}_{c}\right\rangle \simeq \hbar\left(N+\frac{1}{2}\right)
$$

and the expectation value of the Hamiltonian is

$$
\left\langle\hat{H}_{M}\right\rangle \simeq \frac{\mu}{2 R^{2}}\left[\frac{\hbar}{2}\left(x^{2} R^{4}+\frac{1}{x^{2}}+\dot{x}^{2} \frac{R^{4}}{\mu^{2}}\right)+\left\langle\hat{\pi}_{\phi}\right\rangle^{2}+R^{4}\langle\hat{\phi}\rangle^{2}\right] .
$$

On approximating

$$
\left|N_{\lambda}, \tau\right\rangle_{b s} \sim|N, \tau\rangle_{b s}
$$

we have

$$
\begin{aligned}
\left\langle\hat{H}_{M}\right\rangle & \simeq \frac{\mu \hbar}{2}\left(N+\frac{1}{2}\right)\left[\frac{1}{x^{2} R^{2}}+x^{2} R^{2}+\frac{\dot{x}^{2} R^{2}}{\mu^{2}}\right] \\
& \simeq \hbar \mu N\left[1+\frac{\dot{R}^{2}}{2 \mu^{2} R^{2}}\right] \\
& =m_{0}\left[1+\frac{\dot{R}^{2} R_{0}^{3}}{R_{H} R^{2}} \delta^{2}\right] \\
& \equiv m_{0}+\Delta m(\tau),
\end{aligned}
$$

to leading order in $\delta$. This value can be identified with a varying proper mass $m(\tau)$ of the shell. 


\subsection{Consistency conditions and back-reaction}

The previous results were obtained on neglecting the r.h.s.s and the higher WKB order terms in the equations for the matter and gravitational wave-functions. Thus, in order to check the validity of our results, we must estimate the neglected terms and compare them to the corrections we obtain with respect to the classical trajectory in Eq. (3.16). In the following it will be sufficient to consider states $\left|N_{\lambda}, \tau\right\rangle_{b s}$ in the approximation (3.56) and study their evolution for the mean shell radius $R$ greater than the Schwarzschild radius $R_{H}$.

The r.h.s. of the gravitational equation (3.7) can be estimated according to

$$
\begin{aligned}
\frac{\hbar^{2}}{2 M}{ }_{b}\left\langle N\left|\partial_{R}^{2}\right| N\right\rangle_{b} & =-\frac{\hbar^{4} \mu^{2}}{4 M R^{2}} \sum_{P \neq N} \frac{\left\langle N\left|\left(\hat{a}^{\dagger 2}+\hat{a}^{2}\right)\right| P\right\rangle\left\langle P\left|\left(\hat{a}^{\dagger 2}+\hat{a}^{2}\right)\right| N\right\rangle}{\left(E_{N}-E_{P}\right)^{2}} \\
& \simeq-\frac{m^{2}}{4 \mu^{2} M R^{2}}
\end{aligned}
$$

where we used for the time-dependent number of scalar quanta the relation $N(\tau)=m / \hbar \mu$. Therefore, the above correction to the Hamilton-Jacobi equation for gravity is negligible with respect to the matter source $m=\left\langle\hat{H}_{M}\right\rangle$ if

$$
R \gg 1 / \mu
$$

which means that the fluctuations associated with the quantum nature of matter become dominant for a radius of the shell of the order of the Compton wave-length of the constituent quanta (which appears as a general property of quantized models, see also Refs. [10, 11]). It is worth noting that the condition (2.22) which ensures that the width of the shell $d \ll R$ coincides with (3.59) when $R=R_{H}$.

For suitable initial conditions it is possible to have $R>R_{H} \gg 1 / \mu$, so that one may follow the evolution of the shell down to its Schwarzschild radius while using the semiclassical Hamilton-Jacobi equation. However, on using the second line in Eq. (3.57) to estimate $\Delta m$ one finds that the ratio $\rho$ between $\Delta m$ and the quantity in Eq. (3.58) is

$$
\rho<2 M \frac{m_{0}}{m^{2}},
$$

where the maximum (of order 2) is obtained on the horizon. Hence, in order to compute consistently the back-reaction on the trajectory $R(\tau)$ due to an increasing number of quanta, one must include the contribution in Eq. (3.58) into the Hamilton-Jacobi equation. This leads to the equation of motion given below by the first expression in Eq. (3.65).

The r.h.s. of the matter equation (3.7) induces quantum transitions from the (otherwise conserved) state $|N\rangle_{b}$ to states $|L\rangle_{b}$ with $L \neq N$. It is then useful to estimate the projection

$$
\begin{aligned}
{ }_{b}\left\langle L\left|\partial_{R}^{2}\right| N\right\rangle_{b} & =\frac{\hbar^{2} \mu^{2}}{R^{2}} \sum_{P \neq L, N} \frac{\left\langle L\left|\left(\hat{a}^{\dagger 2}+\hat{a}^{2}\right)\right| P\right\rangle\left\langle P\left|\left(\hat{a}^{\dagger 2}+\hat{a}^{2}\right)\right| N\right\rangle}{\left(E_{P}-E_{L}\right)\left(E_{P}-E_{N}\right)} \\
& \simeq \frac{m^{2}}{4 \mu^{2} R^{2}}
\end{aligned}
$$


for $L=N \pm 4$. Since the latter expression leads to a r.h.s. in Eq. (3.7) of the same order of magnitude as Eq. (3.58), it also leads to the same condition, Eq. (3.59), for the validity of the Schrödinger equation (3.18). Further, in Eq. (3.18), if one wishes to include corrections related to $\Delta m$, one must also include the contribution of the r.h.s. of Eq. (3.7) as was done above for the gravitational part. In the following we shall check that, for physically plausible data, $\Delta m \ll m_{0}$ and both the above corrections can be neglected.

Finally we must check the consistency of the WKB approximation used in Eq. (3.17). For the matter equation (3.7) the correction coming from the (neglected) prefactor in the semi-classical wave function $\tilde{\psi}$ is approximatively given by

$$
-\frac{\hbar^{2}}{2 M} \frac{\dot{P}_{R}}{\dot{R} P_{R}} \sim \frac{\hbar^{2} \ddot{R}}{2 M \dot{R}^{2}} \leq \frac{\hbar^{2}}{G M^{2}}
$$

where the last expression is the value on the horizon $R_{H}$. For the gravitational equation the corresponding correction is given by

$$
\frac{\hbar^{2}}{2 M}\left[-\frac{3}{4} \frac{\dot{P}_{R}^{2}}{\dot{R}^{2} P_{R}^{2}}-\frac{\dot{P}_{R} \ddot{R}}{2 \dot{R}^{3} P_{R}}+\frac{\ddot{P}_{R}}{2 \dot{R}^{2} P_{R}}\right] \sim \frac{\hbar^{2}}{2 M}\left[-\frac{5 \ddot{R}^{2}}{4 \dot{R}^{4}}+\frac{1}{2 \dot{R}^{3}} \dddot{R}\right] \leq \frac{\hbar^{2}}{G^{2} M^{3}} .
$$

Both corrections above, when compared with the matter source $m$ are negligible provided

$$
R_{H} \gg \hbar / M
$$

where $\hbar / M$ is the Compton wave-length of the shell as a whole and is much smaller than $1 / \mu$ (see Ref. [10]). Thus if condition (3.59) is satisfied, so is (3.64). Further, the first correction is proportional to $N^{-2}$ and the second one to $N^{-3}$. From the expression for $m$ in Eq. (3.57) one can infer that $\Delta m$ is proportional to the ratio between the Compton wave-length $1 / \mu$ and the radius at which $\Delta m$ is computed (in our case $R>R_{H}$ ). Thus $\Delta m \sim 1 / N$ (on the horizon where $\dot{R}$ is a constant) and both corrections are negligible with respect to $\Delta m$ for $N \gg 1$.

In order to compute the back-reaction on the trajectory of the shell, one can integrate the system of coupled ordinary differential equations governing the semi-classical (Newtonian) trajectory, which we denote by $R_{s}$, and the corresponding proper mass $m$,

$$
\left\{\begin{array}{l}
\dot{R}_{s}^{2}=\frac{G M}{R_{S}}+2\left(1-\frac{m}{M}\right)-\frac{m^{2}}{4 \mu^{2} M^{2} R_{s}^{2}} \\
m=m_{0}\left(1+\frac{\dot{R}_{s}^{2}}{2 \mu^{2} R_{s}^{2}}\right)
\end{array}\right.
$$

The last term in the first equation above corresponds to the quantity in Eq. (3.58). As initial condition we shall set $m(0) \equiv m_{0}=M$. It is then natural to compare the result with $R_{c}$ in Eq. (3.16) which corresponds to the Newtonian limit for the trajectory of a thin shell with $m=m_{0}=M$ constant.

We have computed $R_{s}(\tau)$ and $m(\tau)$ numerically for a set of initial number of quanta $N>10^{38}$, which is the limit required by our model of the shell described in section 2 (see Table 1, the third case is included only as a limiting example). The general picture is that $R_{s}$ collapses slower than $R_{c}$, however the difference $R_{s}-R_{c}$ computed at the proper time when $R_{c}=R_{H}$ is a fraction of $1 / \mu$ and decreases for increasing $N$. Therefore, in our approach, the difference between the semi-classical trajectory $R_{s}$ and $R_{c}$ is negligible in 


\begin{tabular}{|c|c|c|c|c|c|}
\hline $\mathrm{N}$ & $R_{H}$ & $\Delta N$ & $R_{s}-R_{c}$ & $\frac{R_{s}-R_{c}}{R_{c}}$ & $\frac{m^{2}}{8 M^{2}}$ \\
\hline $4 \cdot 10^{40}$ & $500 \frac{1}{\mu}$ & $4 \cdot 10^{34}$ & $2 \cdot 10^{-3} \frac{1}{\mu}$ & $4 \cdot 10^{-6}$ & 0.13 \\
\hline $4 \cdot 10^{39}$ & $50 \frac{1}{\mu}$ & $4 \cdot 10^{35}$ & $2 \cdot 10^{-2} \frac{1}{\mu}$ & $4 \cdot 10^{-4}$ & 0.13 \\
\hline $4 \cdot 10^{38}$ & $5 \frac{1}{\mu}$ & $4 \cdot 10^{36}$ & $2 \cdot 10^{-1} \frac{1}{\mu}$ & $4 \cdot 10^{-2}$ & 0.13 \\
\hline
\end{tabular}

Table 1: Samples of numerical estimates of the effects associated with non-adiabaticity. $N$ is the initial number of particles (with Compton wave-length $1 / \mu=2 \cdot 10^{-14} \mathrm{~cm}$ ) in the shell; $R_{H}$ the corresponding Schwarzschild radius; $\Delta N$ the number of particles produced at the proper time at which the shell coordinate $R_{c}$ reaches the horizon; for the same proper time the relative position of the shell when back-reaction is included is given by $R_{s}-R_{c}$; finally, in the last column we report the ratio between the relativistic correction and the Newtonian potential on the horizon, (see Eq. (3.13)).

all physical situations. Further, relativistic corrections associated to the neglected term $G^{2} m^{2} / 4 R_{s}^{2}$ in the equation of motion (1.7) amount to a deviation from $R_{c}$ of about $13 \%$ on the horizon (see last column in Table 1) which is much greater than the computed relative deviation of the trajectory due to a changing mass or to quantum fluctuations (fifth column in Table 11). Therefore one can safely approximate $R_{s}$ with $R_{c}$ down to the Schwarzschild radius.

The production of matter instead is appreciable. In the second case displayed in Table 1, the number of produced particles $\Delta N=\Delta m / \hbar \mu$ is of order of $10^{-4}$ times the initial mass (see also Fig. 1). In general the data shown in Table 1 are in agreement with the expected behaviour $\Delta N \propto N^{-1}$.

Lastly for $R_{s} \rightarrow 0$ the number $\Delta N$ diverges and our approach breaks down completely since all the consistency conditions are violated.

\section{Conclusions}

Our purpose has been the description of the gravitational collapse of a self-gravitating shell of quantum matter. We have attempted to obtain a "realistic" description of such a shell by considering a bound state of a sufficiently large number of scalar particles. It was heuristically found that it was possible to obtain realistic and "thin" shells.

Since our shell consists of scalar particles it is plausible to assume that the particles, at least initially, will all be in the ground state and form some sort of condensate. Subsequent evolution during the collapse will cause transitions to higher excited bound states which presumably, once a sufficient number of them is formed, will decay collectively through the creation of an additional particle in the ground state. This will lead to a condensate with an increasing number of particles. We have pointed out that this is somewhat analogous to the hadronization process in QCD.

The above approach then led to an effective time dependent Lagrangian for matter. On including the shell Lagrangian and quantizing the matter-gravity system we obtained the corresponding Wheeler-DeWitt equation. On subsequently considering the semiclassical limit for gravity and neglecting fluctuations, we introduce a matter Fock space and reproduce the precedingly obtained Einstein equation for a many scalar particle shell together with the Schrödinger equation for matter. The latter system was then solved using 


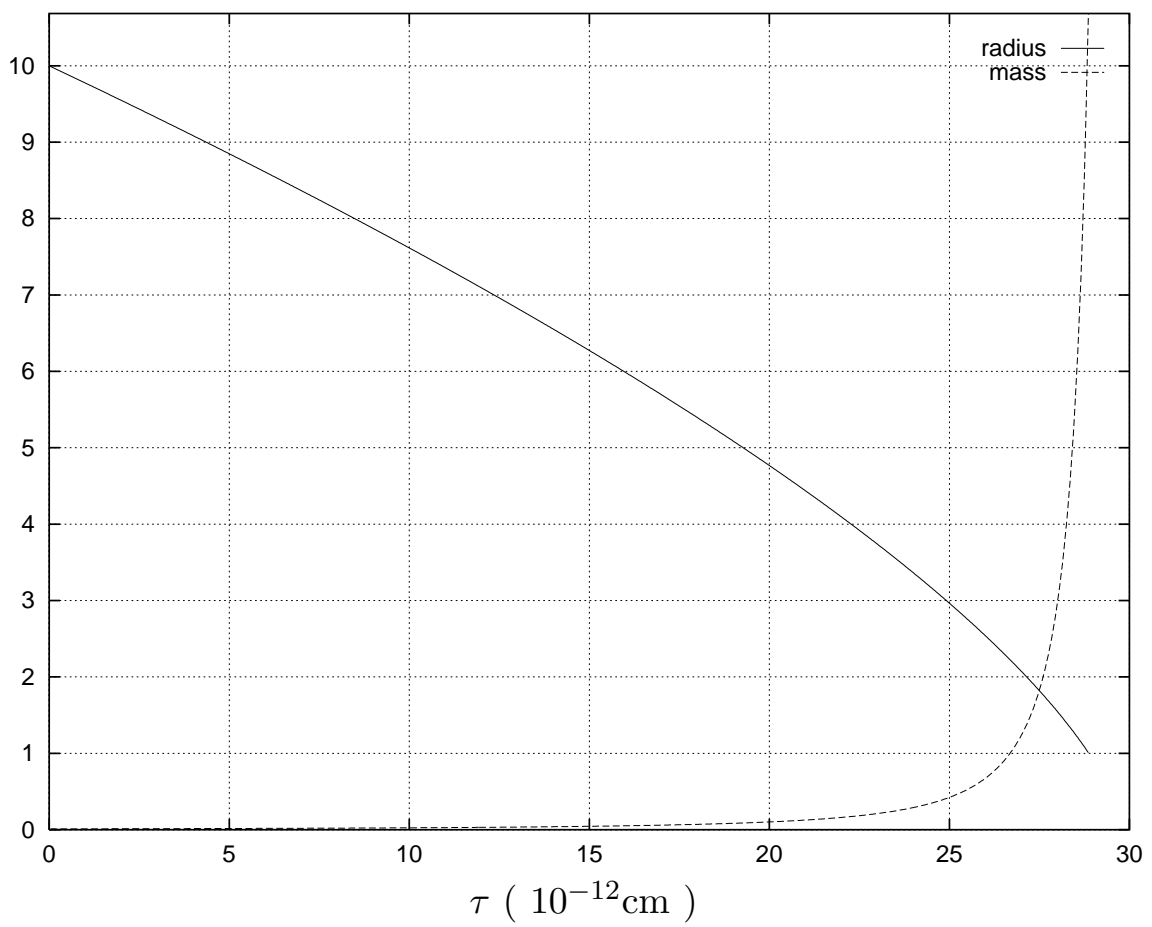

Figure 1: Evolution of the radius $R_{s} / R_{H}$ and relative mass $10^{5}(m-M) / M$ of the shell in proper time for the second case in Table 1 and $R_{s}(0)=10 R_{H}$.

the method of adiabatic invariants.

On analyzing our results, the main conclusion is that, for a suitable number of initial particles constituting the shell, the matter proper energy increases appreciably when the shell radius approaches the horizon. This corresponds to an increase of the number of particles, that is matter creation. Correspondingly, one obtains corrections to the equation of motion for the mean shell radius which lead to a slow down of the collapse. However, for the range of parameter considered, this change does not produce observable effects on the trajectory of the shell. It is clear that suitable conditions should lead to more significant effects, however our approach would then break down.

It would be of interest, within our quantitative approach, to consider an extension to many shells leading to a more general matter distribution in order to study its collapse, again taking into account matter quantum effects. We hope to return to this point.

\section{References}

[1] Tolman R C 1934 Proc. Nat. Acad. Sci. 20169

[2] Oppenheimer J R and Snyder H 1939 Phys. Rev. 56455

[3] Israel W. 1966 Nuovo Cimento B 44 1; Nuovo Cimento B 48463

[4] Singh T P 1998 Gravitational collapse, black holes and naked singularities, preprint gr-qc/9805066 
[5] Arnowitt R Deser S and Misner C W 1962 in Gravitation: an introduction to current research Witten L editor (New York: Wiley)

[6] Lund F 1973 Phys. Rev. D 83253

[7] Brout R 1987 Found. Phys. 17603

[8] Brout R. and Venturi G. 1989 Phys. Rev. D 152436

[9] Bertoni C Finelli F and Venturi G 1996 Class. Quantum Grav. 132375

[10] Casadio R and Venturi G 1996 Class. Quantum Grav. 132715

[11] Casadio R Finelli F and Venturi G 1998, preprint gr-qc/9706074, Class. Quantum Grav. (to be published)

[12] Stephani H 1990 General Relativity (Cambridge: Cambridge Univ. Press)

[13] Goncalves S and Moss I G 1997 Class. Quantum Grav. 142607

[14] Lewis H R and Riesenfeld W B 1969 J. Math. Phys. 101458

[15] We assume the space-time is asymptotically flat and no sources other than the shell itself are present.

[16] Ansoldi S Aurilia A Balbinot R and Spallucci E 1997 Class. Q. Grav 142727

[17] Hajicek P 1998 Phys. Rev. D 57936

[18] Rowe E G P 1987 Eur. Jour. of Phys. 881

[19] Shiff L 1955 Quantum Mechanics (New York: McGraw-Hill)

[20] Klauder J R 1996 J. Phys. A 29293

[21] Bellomo P and Stroud C R Dispersion of Klauder's temporally stable coherent states for the hydrogen atom, preprint quant-ph/9804074

[22] Again one has an analogy with hadronic physics in which an excited hadron (long string) decays into lighter ones (short strings).

[23] Nambu J and Bindu Bambah 1982 Phys. Rev. D 262871

[24] Lewis H R 1968 J. Math. Phys. 91976 